\title{
Qualidade de vida dos pacientes portadores de síndrome de Stevens-Johnson
}

\author{
Quality oflife in patients with Stevens-Johnson syndrome
}

\author{
Ruth Nogueira ${ }^{1}$ \\ Maciel Franca ${ }^{2}$ \\ Marcelo G. Lobato ${ }^{3}$ \\ Ricardo Belfort ${ }^{4}$ \\ Camila B. Souza ${ }^{5}$ \\ José Álvaro P. Gomes ${ }^{6}$
}

\section{RESUMO}

Objetivo: Avaliar a qualidade de vida dos pacientes portadores de síndrome de Stevens-Johnson. Métodos: Foram avaliados 14 pacientes com SSJ no período de 1998 e 1999 no Setor de Córnea do Departamento de Oftalmologia da UNIFESP.Empregou-se o questionário S. F. 36-pesquisa em saúde. A pontuação indica melhor condição de saúde. A mesma avaliação foi repetida quatro vezes em intervalo de três meses. Resultados: As médias das médias das 4 avaliações dos 14 pacientes apresentaram importante redução dos seus valores em todos os subitens do S.F. 36 $(41,04 \pm 22,38$ para aspectos físicos, $53,82 \pm 22,82$ para aspectos sociais, $26,24 \pm 22,46$ para aspectos emocionais, $34,95 \pm 25,45$ para capacidade funcional, 42,03 $\pm 31,85$ para dor, $46,33 \pm 18,37$ para vitalidade, $46,18 \pm 24,83$ para saúde mental e 50,26 $\pm 16,29$ para saúde geral). Conclusão: O questionário S.F. 36 representa método adequado de avaliação de aspectos físicos e emocionais em pacientes com baixa de acuidade visual, que nos permitiu detectar comprometimento importante nas funções básicas dos pacientes com SSJ. Mais pacientes com maior tempo de seguimento são necessários para aprimorar a análise de qualidade de vida nesses pacientes e avaliar os efeitos do acompanhamento psicológico.

Descritores: Síndrome de Stevens-Johnson; Qualidade de vida; Questionários
Trabalho realizado no Departamento de Oftalmologia da Universidade Federal de São Paulo - UNIFESP.

${ }_{1}^{1}$ Psicóloga do Setor de Psicologia aplicada à Oftalmologia do Departamento de Oftalmologia da Universidade Federal de São Paulo - UNIFESP.

${ }^{2}$ Ex-estagiário do Setor de Doenças Externas e Córnea do Departamento de Oftalmologia da Universidade Federal de São Paulo - UNIFESP.

${ }^{3}$ Psicólogo e Técnico do Setor de Cirurgia Experimental do Departamento de Oftalmologia da Universidade Federal de São Paulo - UNIFESP.

${ }^{4}$ Doutor em Ciências Visuais e Chefe do Setor de Psicologia aplicada à Oftalmologia do Departamento de Oftalmologia da Universidade Federal de São Paulo - UNIFESP.

${ }^{5}$ Psicóloga do Setor de Psicologia aplicada à Oftalmologia do Departamento de Oftalmologia da Universidade Federal de São Paulo - UNIFESP e da UMESP.

${ }^{6}$ Doutor em Medicina e Colaborador do Setor de Doenças Externas e Córnea do Depto. de Oftalmologia da Universidade Federal de São Paulo - UNIFESP; Coordenador da Residência de Oftalmologia da Universidade de Alfenas/MG (UNIFENAS)

Endereço para correspondência: R. Sabará, 566 apto. 212 - São Paulo (SP) CEP 01239-010.

E-mail: japgomes@uol.com.br

Recebemos para publicação em 09.03.2001 Aceito para publicação em 11.09.2002

Nota Editorial: Pela análise deste trabalho e por sua anuência sobre a divulgação desta nota, agradecemos ao Dr. Eduardo Melani Rocha.

\section{INTRODUÇÃO}

A síndrome de Stevens-Johnson (SSJ) pode ser definida como uma afecção inflamatória aguda, febril e autolimitada, com duração aproximada de duas a quatro semanas, que afeta a pele e a membrana mucosa ${ }^{(1-4)}$. A síndrome inicia-se geralmente após o uso de medicações ou ocorrência de infecções e provavelmente apresenta etiopatogenia autoimune ${ }^{(2-5)}$.

O quadro ocular é caracterizado por uma conjuntivite purulenta catarral bilateral, membranosa ou pseudomembranosa ${ }^{(1)}$. Na fase crônica, a maioria dos pacientes apresenta numerosas alterações da superfície ocular que podem comprometer a acuidade visual, destacando simbléfaro, entrópio, ectrópio, triquíase, olho seco, conjuntivalização e queratinização corneana ${ }^{(6-7)}$.

A incidência de SSJ está estimada entre 1 a 6 casos por um milhão de habitantes ${ }^{(2-5)}$. Apesar de rara, esta doença gera um forte impacto emocional, social e econômico, pois se trata de uma entidade crônica que leva potencialmente a cegueira de pacientes jovens.

O objetivo desse trabalho é avaliar a qualidade de vida dos pacientes com SSJ nos aspectos físicos, sociais, emocionais, capacidade funcional, saúde mental e estado geral de saúde. 


\section{MÉTODOS}

Foram avaliados 14 pacientes com SSJ, no período de setembro de 1998 a setembro de 1999, no setor de Doenças Externas e Córnea do Departamento de Oftalmologia da Universidade Federal de São Paulo. Os pacientes foram convocados pela psicóloga que, após esclarecimentos sobre a natureza da pesquisa, solicitou que respondessem individualmente o questionário S.F.36 - pesquisa em saúde. Este questionário é considerado um índice de percepção do status geral de saúde que incorpora padrões comportamentais. O S.F. 36 foi traduzido e validado para a língua portuguesa em $1997^{(8)}$. O questionário compõe-se de oito sub-itens (sub-escalas): capacidade funcional, aspectos físicos, dor, estado geral de saúde, vitalidade, aspectos sociais, emocionais e saúde mental. Os dados brutos obtidos nas respostas são convertidos em dados ponderados, que são transformados em valores de 0 a $100(0=$ pior $100=$ melhor $)$ pela fórmula de cálculo: ${ }^{(8)}$

Valor ponderado - limite inferior x 100 Variação (score range)

A mesma avaliação foi repetida quatro vezes para cada um dos pacientes em um intervalo de três meses. As perguntas foram feitas oralmente, devido à baixa de acuidade visual dos pacientes, e cada sessão durava aproximadamente 40 minutos. Após responderem ao S.F. 36, os pacientes foram submetidos a duas entrevistas psicológicas semanais. Depois dessas duas entrevistas, os pacientes, caso concordassem, eram encaminhados para psicoterapia grupal breve.

\section{RESULTADOS}

Dos 14 pacientes avaliados, $9(64 \%)$ eram do sexo feminino e $5(36 \%)$ eram do sexo masculino. $14 \%$ eram de raça negra, $36 \%$ pardos, $7 \%$ amarelos e $43 \%$ brancos. A idade variou entre 17 e 75 anos, perfazendo uma média de 29 anos.

A média de idade dos pacientes examinados foi de 34,5 anos (17-74 anos) e o tempo médio de doença foi de 135,9 meses (4 - 480 meses). Os dados demográficos, do quadro de SSJ e do acometimento ocular dos pacientes encontram-se na tabela 1 .

As médias das médias dos índices do S.F. 36 das 4 avaliações dos 14 pacientes foram de $41,04 \pm 22,38$ para aspectos

\begin{tabular}{|c|c|c|c|c|c|c|c|}
\hline $\mathbf{N}$ & $\begin{array}{l}\text { Sexo/ldade } \\
\text { (anos) }\end{array}$ & $\begin{array}{l}\text { Início da SSJ } \\
\text { (anos) }\end{array}$ & $\begin{array}{c}\text { Fator } \\
\text { desencadeante }\end{array}$ & AV CC & $\begin{array}{l}\text { Severidade da } \\
\text { doença ocular }{ }^{14}\end{array}$ & $\begin{array}{l}\text { Schirmer I } \\
(\mathrm{mm})\end{array}$ & Cirurgia prévia \\
\hline \multirow[t]{2}{*}{1} & $\mathrm{~F} / 26$ & 3 & Perlutan & $O D=20 / 100$ & $\mathrm{OD}=\mathrm{IIC}$ & $\mathrm{OD}=2$ & Tp de MA \\
\hline & & & & $O E=20 / 200$ & $\mathrm{OE}=\| \mathrm{IC}$ & $\mathrm{OE}=0$ & (OD e OE) \\
\hline \multirow[t]{2}{*}{2} & $\mathrm{M} / 46$ & 6 & Dipirona & $\mathrm{OD}=\mathrm{MM}$ & $O D=11 c$ & $\mathrm{OD}=0$ & Tx de córnea, ML, \\
\hline & & & & $\mathrm{OE}=\mathrm{MM}$ & $\mathrm{OE}=I \mathrm{IC}$ & $\mathrm{OE}=0$ & $M A$ e $L(O D$ e $O E)$ \\
\hline \multirow[t]{2}{*}{3} & $\mathrm{M} / 48$ & 22 & Sulfa & $\mathrm{OD}=\mathrm{Cd}$ & $O D=\| l c$ & $O D=5$ & - \\
\hline & & & & $\mathrm{OE}=20 / 30$ & $\mathrm{OE}=\mathrm{lc}$ & $\mathrm{OE}=24$ & \\
\hline \multirow[t]{2}{*}{4} & F/68 & 40 & Hidantal & $\mathrm{OD}=\mathrm{MM}$ & $O D=11 c$ & $\mathrm{OD}=0$ & Tx de córnea, $T x$ \\
\hline & & & & $\mathrm{OE}=\mathrm{MM}$ & $\mathrm{OE}=\| \mathrm{IC}$ & $\mathrm{OE}=0$ & de $M L(O D$ e $O E)$ \\
\hline \multirow[t]{2}{*}{5} & $F / 18$ & 1,2 & Dipirona & $\mathrm{OD}=\mathrm{MM}$ & $\mathrm{OD}=\| \mathrm{IC}$ & $O D=20$ & Cola, "Patch" de \\
\hline & & & & $O E=20 / 20$ & $\mathrm{OE}=\mathrm{lc}$ & $\mathrm{OE}=12$ & córnea OD \\
\hline \multirow[t]{2}{*}{6} & $M / 62$ & 11 & Dipirona & $\mathrm{OD}=\mathrm{SPL}$ & $O D=I I C$ & $\mathrm{OD}=5$ & Tp de ML, Tarso \\
\hline & & & & $\mathrm{OE}=\mathrm{MM}$ & $\mathrm{OE}=1 \mathrm{Ic}$ & $\mathrm{OE}=5$ & (OD e OE) \\
\hline \multirow[t]{2}{*}{7} & $F / 32$ & 6 & Dipirona & $\mathrm{OD}=\mathrm{MM}$ & $O D=\| l c$ & $O D=10$ & Tp de ML, Tarso \\
\hline & & & & $\mathrm{OE}=\mathrm{Cd}$ & $\mathrm{OE}=\| \mathrm{IC}$ & $\mathrm{OE}=35$ & (OD e OE) \\
\hline \multirow[t]{2}{*}{8} & $F / 18$ & 5 & Carbamazepina/ & $\mathrm{OD}=20 / 60$ & $\mathrm{OD}=11 \mathrm{c}$ & $\mathrm{OD}=7$ & - \\
\hline & & & Dipirona & $\mathrm{OE}=20 / 80$ & $\mathrm{OE}=1 \mathrm{Ic}$ & $\mathrm{OE}=3$ & \\
\hline \multirow[t]{2}{*}{9} & $F / 28$ & 8 & Dipirona & $\mathrm{OD}=\mathrm{PL}$ & $O D=11 \mathrm{c}$ & $O D=7$ & Tarso OD \\
\hline & & & & $O E=20 / 40$ & $\mathrm{OE}=\mathrm{Ic}$ & $\mathrm{OE}=10$ & \\
\hline \multirow[t]{2}{*}{10} & $\mathrm{~F} / 30$ & 0,4 & Diclofenaco & $O D=20 / 400$ & $O D=11 c$ & $O D=5$ & - \\
\hline & & & & $O E=20 / 400$ & $\mathrm{OE}=\| \mathrm{Ic}$ & $\mathrm{OE}=5$ & \\
\hline \multirow[t]{2}{*}{11} & $F / 25$ & 5 & Penicilina & $\mathrm{OD}=\mathrm{Cd}$ & $O D=I C$ & $O D=10$ & - \\
\hline & & & & $\mathrm{OE}=20 / 20$ & $\mathrm{OE}=\mathrm{Ic}$ & $\mathrm{OE}=20$ & \\
\hline \multirow[t]{2}{*}{12} & $\mathrm{M} / 26$ & 21 & Dipirona & $\mathrm{OD}=\mathrm{MM}$ & $O D=11 c$ & $\mathrm{OD}=0$ & $\mathrm{Tp} M A+L$ OD \\
\hline & & & & $\mathrm{OE}=\mathrm{PL}$ & $\mathrm{OE}=\| \mathrm{IC}$ & $\mathrm{OE}=0$ & \\
\hline \multirow[t]{2}{*}{13} & $M / 58$ & 15 & $?$ & $O D=20 / 100$ & $O D=I c$ & $O D=0$ & - \\
\hline & & & & $\mathrm{OE}=\mathrm{Cd}$ & $\mathrm{OE}=I \mathrm{IC}$ & $\mathrm{OE}=5$ & \\
\hline \multirow[t]{2}{*}{14} & $\mathrm{~F} / 17$ & 10 & Dipirona & $\mathrm{OD}=\mathrm{PL}$ & $O D=\| \mathrm{C}$ & $\mathrm{OD}=0$ & - \\
\hline & & & & $\mathrm{OE}=20 / 200$ & $\mathrm{OE}=\mathrm{lc}$ & $\mathrm{OE}=3$ & \\
\hline
\end{tabular}


físicos, $53,82 \pm 22,82$ para aspectos sociais, $26,24 \pm 22,46$ para aspectos emocionais, $34,95 \pm 25,45$ para capacidade funcional, $42,03 \pm 31,85$ para dor, $46,33 \pm 18,37$ para vitalidade, $46,18 \pm 24,83$

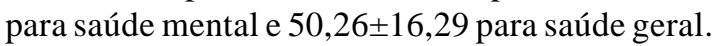

As médias dos resultados do questionário S.F. 36 referentes aos parâmetros atividades físicas, social, emocional, funcional, vitalidade, dor, saúde mental e estado geral de saúde dos pacientes em estudo nas 4 avaliações encontram-se expressos no gráfico 1 .

\section{DISCUSSÃO}

A SSJ é uma doença com seqüelas oculares que causam baixa de visão e sintomas de desconforto, o que induz grande transtorno psicológico, social e econômico numa população com média de idade de aproximadamente 30 anos. Esta faixa etária corresponde a fase mais produtiva do indivíduo, o que causa extrema ansiedade e depressão nesses pacientes. Daí, a necessidade do apoio psicológico contínuo.

O questionário S.F. 36 foi introduzido para avaliar a qualidade de vida de pacientes portadores de deficiência visual, pois verifica diversos aspectos físicos e emocionais que podem estar comprometidos em função desta perda ${ }^{(9-14)}$. Baixos índices de todos os sub-itens do S.F. 36 (variação na média aproximada entre 60 a $75 \%$ ) foram encontrados em pacientes com baixa visão candidatos a transplante de córnea ${ }^{(12)}$. Pacientes portadores de glaucoma também apresentaram comprometimento importante de qualidade de vida pelo S.F. 36, embora em menor escala que nos candidatos a transplante de

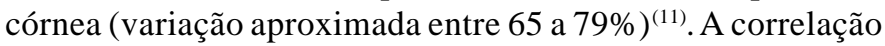
entre acuidade visual e qualidade de vida pelo S.F. 36 apresentou-se válida apenas no parâmetro aspecto físico ${ }^{(7)}$.

Neste trabalho, procuramos avaliar os aspectos físicos, sociais, emocionais, capacidade funcional, dor, saúde mental e estado geral de saúde em pacientes com SSJ com seqüelas oculares atendidos em um serviço terciário de oftalmologia.

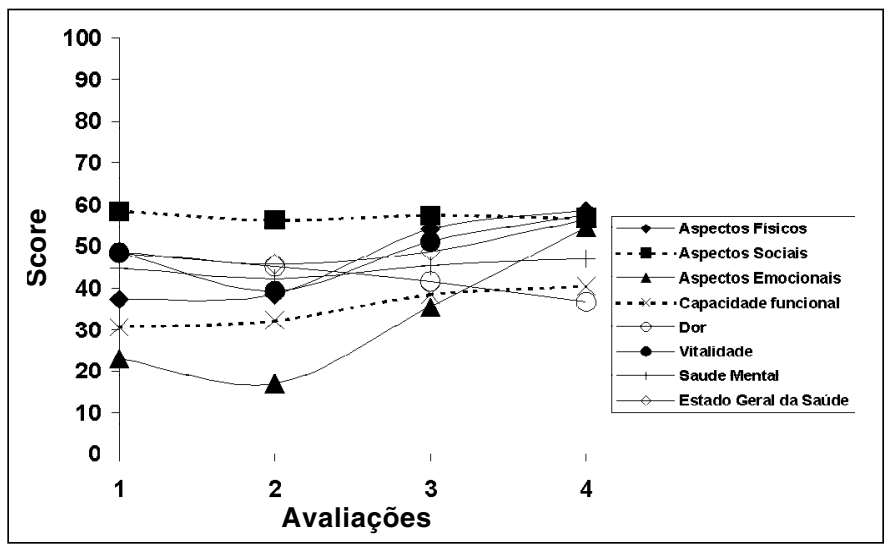

Gráfico 1 - Comparação das médias dos resultados dos aspectos físicos, sociais, emocionais, capacidade funcional, vitalidade, dor, saúde mental e estado geral de saúde do questionário S.F. 36 obtidas nas quatro avaliações realizadas com os 14 pacientes de SSJ entrevistados
Esses dados ainda não foram relatados na literatura nacional e têm sido pouco explorados na literatura internacional ${ }^{(5)}$. Encontramos importante diminuição dos índices de várias funções, destacando-se os aspectos físico, funcional, dor e, principalmente, emocional (todos abaixo de $50 \%$ e o último ao redor de $27 \%$ ). Resultados similares foram observados na prática clínica em pacientes HIV+ portadores de retinopatia por citomegalovirus (R.N., comunicação pessoal).

Devido ao pequeno número de casos e perda de seguimento de 4 pacientes, não foi possível realizar análise estatística dos resultados. Além disso, há grande variação das respostas do questionário S.F. 36 entre os pacientes que se correlaciona, na maioria das vezes, com o acometimento da acuidade visual. Importante observar que houve melhora dos índices do S.F. 36 em relação à capacidade funcional, aspectos físicos e vitalidade no decorrer das avaliações, diferentemente do que ocorreu com dor. Talvez esse achado reflita a importância do início do acompanhamento psicológico desses pacientes.

É importante salientar que os pacientes necessitam de tratamento clínico contínuo, como reposição de lágrimas artificiais e, muitas vezes, intervenções cirúrgicas ${ }^{(6,14)}$. Portanto, é fundamental que estejam conscientes da importância de sua colaboração no tratamento, muitas vezes fadado ao insucesso. O questionário S.F. 36 ajudou-nos a compreender melhor as necessidades dos pacientes com SSJ, o que refletiu positivamente no seguimento psicológico e na dinâmica do grupo.

Em conclusão, o questionário S.F. 36 representa um método adequado de avaliação de aspectos físicos e emocionais em pacientes com baixa de acuidade visual que nos permitiu detectar comprometimentos importantes nas funções básicas dos pacientes com SSJ. Mais pacientes com maior tempo de seguimento são necessários para aprimorar a análise de qualidade de vida nesses pacientes e avaliar os efeitos do acompanhamento psicológico.

\section{ABSTRACT}

Purpose: To assess the quality of life in patients with Stevens-Johnson syndrome (SJS). Methods: Fourteen patients with SJS were evaluated between 1998 and 1999 at the External Disease and Cornea Service of the Dept. of Ophthalmology of UNIFESP. We used a S.F. 36 questionnaire. The score for the seven subitems was given based on described formula. High scores mean a better life condition. The same questionnaire was repeated 4 times in a 3 months period. Results: The means of the means of the 4 questionnaire values for the 14 patients were decreased in all S.F. 36 subitems $(41.04 \pm 22.38$ for physical aspects, $53.82 \pm 22.82$ for social aspects, $26.24 \pm 22.46$ for emotional aspects, $34.95 \pm 25.45$ for functional aspects, $42.03 \pm$ 31.85 for pain, $46.33 \pm 18.37$ for vitality, $46.18 \pm 24.83$ for mental health and 50.26 \pm 16.29 for general health). Conclusions: The S.F. 36 questionnaire is an important method to assess physical and emotional aspects of patients with low vision. It allowed us to detect important limitations in patients with SJS. 
More patients with a larger follow up are necessary to improve our analysis and further evaluate the effects of psychological intervention.

Keywords: Stevens-Johnson syndrome; Quality of life; Questionnaires

\section{REFERÊNCIAS}

1. Stewart MG, Duncan NO, Franklin DJ, Friedman EM, Sulek M. Head and neck manifestations of erythema multiform in children. Otolaryngol Head Neck Surg 1994;111:236-42.

2. Power WJ, Ghoraishi M, Merayo-Lloves J, Neves RA, Foster CS. Analysis of the acute ophthalmic manifestations of the erythema multiforme / Stevens Johnson Syndrome / toxic epidermal necrolysis disease spectrum. Ophthalmology 1995;102:1669-76.

3. Manden SM. Serious and life threatening drug eruptions. Am Fam Physician 1995;51:1865-72.

4. Shimazaki J, Shimmura S, Fujishima H, Tsubota K. Association of preoperative tear function with surgical outcome in severe Stevens-Johnson Syndrome. Ophthalmology 2000;107:1518-23.

5. Kaufman DW. Epidemiologic approaches to the study of toxic epidermal necrolysis. J. Invest Dermatol 1994;102:31S-33S.
6. Gomes JAP. Atualização no tratamento das ceratoconjuntivites cicatriciais. Arq Bras. Oftalmol 2000;63:91-96.

7. Lee PP, Whitcup SM, Hays RD, Spritzer K, Javitt J. The relationship between visual acuity and functioning and well-being among diabetics. Qual Life Res 1995,4;319-23.

8. Ciconelli RM. Tradução para o português e validação do questionário genérico de avaliação de qualidade de vida "medical outcomes study 36-item sort form health survey (SF36)" [Tese ]. São Paulo: Universidade Federal de São Paulo; 1997.

9. Ware JE, Sherbourne CD. The MOS 36 - item short-form health survey (SF 36) -1. Conceptual framework and item selection. Med Care 1992;30:473-83.

10. Dauphinee SW, Gauthier L, Gandek B, Magnan L, Pierre U. Readying a US measure of health, the SF-36, for use in Canada. Clin Invest Med 1997; 20:224-38.

11. Wilson MR, Coleman AL, Yu F, Bing EG, Sasaki IF, Berlin K, Winters J, Lai A. Functional status and well-being in patients with glaucoma as measured by the Medical Outcomes Study Short Form - 36 questionnaire. Ophthalmology 1998;105:2112-6.

12. Boisjoly H, Gresset J, Fontaine N, Charest M, Brunette I, LeFrançois M, Deschenes J, Bazin R, Laughrea PA, Dube I. The VF-14 index of functional visual impairment in candidates for a corneal graft. Am J Ophthalmol 1999;128:38-44.

13. McHorney CA, Ware Je Jr., Raczek AE. The MOS 36 item short form health Survey (SF-36): Psychometric and clinical tests of validity in measuring physical and mental health constructs. Med Care 1993,31;247-63.

14. Schwartz GS, Gomes JAP, Holland EJ. Preoperative staging of disease severity. In: Holland EJ \& Mannis MJ eds. Ocular surface disease-Medical and surgical management. New York: Springer-Verlag; 2001. p.158-67 